\title{
Les trotskystes grecs pendant la seconde guerre Mondiale
}

\section{Alexis Hen}

\section{OpenEdition}

1 Journals

Édition électronique

URL : https://journals.openedition.org/ceb/756

DOI : $10.4000 /$ ceb.756

ISSN : 2261-4184

Éditeur

INALCO

Édition imprimée

Date de publication : 30 mars 2011

Pagination : 71-84

ISBN : 978-2-85831-189-7

ISSN : 0290-7402

\section{Référence électronique}

Alexis Hen, «Les trotskystes grecs pendant la seconde guerre Mondiale », Cahiers balkaniques [En ligne], 38-39 | 2011, mis en ligne le 29 novembre 2011, consulté le 06 juillet 2021. URL : http:// journals.openedition.org/ceb/756; DOI : https://doi.org/10.4000/ceb.756

Ce document a été généré automatiquement le 6 juillet 2021.

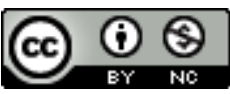

Cahiers balkaniques est mis à disposition selon les termes de la Licence Creative Commons Attribution - Pas d'Utilisation Commerciale 4.0 International. 


\title{
Les trotskystes grecs pendant la seconde guerre Mondiale
}

\author{
Alexis Hen
}

\section{Origines du trotskysme grec}

1 Les divisions du mouvement trotskyste rendent son histoire complexe, et pour la comprendre, il faut remonter aux origines du communisme grec, dont est issue la mouvance trotskyste. Le premier véritable parti ouvrier grec n'a été fondé que fin 1918. Il y eut de nombreuses luttes accompagnées de scissions lors de ses deux premiers congrès, jusqu'à ce que ce parti, le SEK (Parti ouvrier socialiste), adhérât à la Troisième Internationale (communiste) de Lénine et Trotsky en 1920. Certains des cadres fondateurs de l'opposition de gauche trotskyste ultérieure, comme Tzoulati, quittèrent une première fois le SEK en 1919, car ils le trouvaient réformiste, en prenant comme preuve ses atermoiements à passer de la Deuxième Internationale, socialiste, à la Troisième. Ils revinrent au SEK fin 1920, après son adhésion à l'Internationale communiste.

2 Les mêmes créèrent, en 1923, des groupes d'éducation marxiste basés sur la traduction, l'étude et la diffusion des textes classiques tirés des archives du marxisme, d'où leur dénomination d'archéiomarxistes. Ces groupes étaient conçus comme des groupes d'éducation pour les militants du Parti communiste de Grèce, le KKE, mais ils furent exclus de ce parti dès l'année suivante. La séparation des archéiomarxistes avec le KKE est donc antérieure à l'exclusion de Trotsky du PC d'Union soviétique, qui n'intervint que fin 1927. Ce n'est qu'en 1930 que les archéiomarxistes rejoignirent formellement l'Opposition de Gauche Internationale de Trotsky.

3 Au début des années 1930, le KKE était en crise, en liaison avec la stalinisation du parti parallèlement à celle de l'Internationale communiste. À l'inverse, les archéiomarxistes étaient à leur apogée. Ils avaient plusieurs centaines de membres et avaient commencé à s'implanter dans les syndicats. Ils étaient pratiquement la section la plus nombreuse 
de l'Opposition de Gauche Internationale trotskyste; le rapport de forces numérique entre communistes et trotskystes était en Grèce de trois pour un seulement.

Mais Trotsky, qui connaissait très bien les Balkans (il avait été correspondant de guerre pour un quotidien ukrainien pendant les guerres balkaniques), était préoccupé par certains aspects de l'archéiomarxisme. Il revint plusieurs fois sur la relative indifférence des archéiomarxistes à la question nationale macédonienne lors de ses entretiens avec des représentants du mouvement début 1932 ; ceux-ci prenaient pour argent comptant les chiffres fournis par le gouvernement grec qui prétendait que $90 \%$ de la population de Macédoine grecque était maintenant ethniquement grecque suite aux purifications ethniques (les "échanges de populations" obligatoires ou "volontaires ») de 1923. Trotsky en doutait.

5 Les trotskystes grecs étaient relativement indifférents à la question nationale, ce qui allait les prédisposer, plus tard, quand ce fut aux Grecs d'être victimes d'une certaine oppression nationale sous l'occupation nazie (et italo-bulgare), à l'ignorer et à renvoyer dos à dos la résistance, y compris celle sous contrôle communiste, et l'occupant nazi.

6 Si l'on remonte plus loin dans le temps, aux origines du communisme grec, lors de la campagne d'Asie mineure, au début des années 1920, on remarque que nombre de futurs dirigeants communistes étaient au front. Certains, parmi eux, voyaient dans cette campagne une guerre de libération nationale grecque, comme Zakhariadis (le futur dirigeant du KKE stalinisé), alors que des militants comme Pouliopoulos, qui allaient plus tard se déclarer pour Trotsky, étaient contre cette guerre et exigeaient le rapatriement immédiat des troupes. Il faut rappeler toutefois que Lénine n'était pas simplement opposé à cette guerre : il y voyait une guerre de libération nationale turque et soutenait les forces de Mustapha Kemal contre les impérialistes britanniques, et initialement français, qui voulaient dépecer la Turquie à l'aide de troupes grecques.

7 En 1933, la victoire de Hitler en Allemagne fut rendue possible par la trahison de la social-démocratie, mais aussi du Parti communiste allemand stalinien, qui ne fit rien pour stopper les nazis. Lorsque toute l'Internationale communiste refusa de mettre en cause et même de discuter ce qu'avait fait le PC, Trotsky considéra l'Internationale communiste comme finie pour la révolution, et s'orienta vers la construction d'une Quatrième Internationale. Mais une bonne partie des archéiomarxistes refusèrent ces conclusions et firent scission du courant trotskyste international en 1934. Une fois cette séparation effectuée, ils périclitèrent.

8 Dans la deuxième partie des années 1930 toute une série de groupes, y compris des archéiomarxistes qui avaient refusé de rompre avec Trotsky, se sont progressivement regroupés autour de la figure de Pantelis Pouliopoulos. Pouliopoulos avait été secrétaire général du KKE entre 1925 et 1927. Il était allé en prison sous la dictature de Pangalos, et avait courageusement défendu lors de son procès la position officielle de l'Internationale communiste pour une " Macédoine et Thrace unies et indépendantes ", alors qu'il trouvait ce mot d'ordre erroné, du fait notamment de l'inexistence d'une nation thrace. Cela dit, contrairement au KKE qui allait officiellement renoncer à ce mot d'ordre vers 1935 avec la consolidation de la politique de « front populaire » (avec la fraction vénizéliste de l'éventail politique bourgeois), Pouliopoulos défendit toujours le droit d'autodétermination pour les Slavo-Macédoniens.

9 Il devint oppositionnel dans le KKE vers cette époque, sur la question nationale, mais aussi parce qu'il critiquait le mot d'ordre de « démocratie de gauche » qui avait été mis en avant par le parti pendant qu'il était en prison. Il démissionna de son poste en 
septembre 1926 et fut exclu, fin 1927, du KKE, malgré le soutien dont il disposait dans l'organisation du Pirée. Une fois exclu, il refusa de fusionner avec les archéiomarxistes, qui, rappelons-le, allaient former la section officielle de l'Opposition de Gauche Internationale de Trotsky, et il poursuivit un cours indépendant pendant près de dix ans avant de se rapprocher de la Quatrième Internationale à partir de 1935 (il s'était lui-même initialement opposé au tournant de Trotsky vers la Quatrième Internationale en 1933).

10 Les archéiomarxistes qui avaient maintenu les liens avec Trotsky étaient dirigés par Georges Vitsoris. Quand Vitsoris quitta la Grèce pour la France, en 1937, pour échapper à la dictature de Metaxas, Ayis Stinas, un jeune dirigeant du KKE qui en avait été exclu en 1930, prit de plus en plus d'influence dans l'organisation des archéiomarxistes restés avec Trotsky. Il en devint le dirigeant après le départ de Vitsoris et sa politique s'éloigna de plus en plus de celle des trotskystes. Dès 1937, il commença à rompre avec la définition trotskyste de l'URSS comme "État ouvrier dégénéré ». Pendant la guerre les groupes de Pouliopoulos et de Stinas allaient demeurer profondément divisés sur cette question.

11 Entre ces deux groupes, il y eut toute une série de scissions et de regroupements, allant d'un groupe à l'autre, parfois revenant au KKE. Lors de la conférence de fondation de la Quatrième Internationale en 1938, en région parisienne, le groupe de Stinas et celui de Pouliopoulos étaient tous deux représentés, et la conférence préconisa la fusion des deux groupes. Elle n'eut, en fait, pas lieu avant 1946 et fut éphémère. Donc, les trotskystes abordèrent divisés le coup d'État de Metaxas d'août 1936, et ils le demeurèrent.

\section{Les débats dans les camps de concentration de Metaxas}

Dans les dix-huit mois suivant l'instauration de la dictature de Metaxas, une grande partie des trotskystes, y compris Stinas et Pouliopoulos, furent arrêtés et envoyés dans des camps de concentration. Là, les deux groupes menèrent de longues discussions dans la perspective de l'unification demandée par la Quatrième Internationale. Ils avaient des divergences sur la situation en Grèce et le bilan des années 1935-1936 notamment, et, de façon plus nette encore, sur l'attitude à avoir vis-à-vis de l'Union soviétique.

Stinas, dont l'organisation représentait la continuité de la section archéiomarxistetrotskyste, considérait qu'il y avait eu une situation révolutionnaire en Grèce entre la tentative de coup d'État vénizéliste de mars 1935 et le soulèvement ouvrier de Salonique de mai 1936, et que l'échec de celui-ci avait conduit à un reflux irréversible de la vague révolutionnaire, ouvrant la voie à la dictature de Metaxas, qu'il considérait comme fasciste. Pouliopoulos, lui, considérait le régime de Metaxas comme bonapartiste, donc sans une base de masse dans la petite bourgeoisie comme dans le cas d'un régime fasciste, et niait que la classe ouvrière grecque eût subi une défaite catastrophique comparable à celle du prolétariat allemand en 1933. Il est à noter que Trotsky lui-même, avant qu'il fût assassiné en août 1940, avait caractérisé le régime de Vichy, dans ses premiers mois, de « bonapartiste sénile » et non de fasciste.

Pouliopoulos avait prôné la neutralité lors du coup d'État de 1935, alors que Vitsoris et Stinas avaient au contraire préconisé une action de classe en 1935, indépendamment 
des populistes et des vénizélistes. Le groupe de Vitsoris/Stinas fut confronté à une opposition interne plus attentiste, qui finit par faire sécession et renforcer le groupe de Pouliopoulos. À l'inverse, Pouliopoulos était prêt l'année suivante à faire un front unique avec les vénizélistes contre le coup d'État de Metaxas, pendant qu'en 1936 Stinas refusait un tel bloc qu'il voyait comme une compromission politique avec les vénizélistes. Le bilan de cette période fut l'objet d'âpres débats entre les deux groupes dans les camps.

Les deux groupes étaient d'accord pour refuser de soutenir la Grèce dans le conflit mondial, y compris lorsqu'elle fut attaquée par l'Italie de Mussolini. Cela contraste fortement avec le KKE qui, malgré des réticences initiales à la base, se résolut à soutenir le « non » de Metaxas à l'ultimatum de Mussolini. La position trotskyste était conforme à l'attitude marxiste qui interprétait les événements comme un conflit entre les impérialistes de l'Entente et ceux de l'Axe, où la Grèce n'était qu'un simple pion. Mais les trotskystes grecs considéraient la Grèce elle-même comme impérialiste, la mettant ainsi sur le même pied que l'Italie. Cela allait conduire les deux groupes à nier les éléments d'oppression nationale des Grecs pendant l'occupation italo-bulgaroallemande.

Stinas et Pouliopoulos étaient d'accord pour s'opposer au nationalisme grec du KKE. Pourtant, les deux groupes avaient une divergence essentielle concernant le caractère de l'Union soviétique et, en conséquence, l'attitude à avoir dans la guerre, notamment vis-à-vis de ce pays. Cette question était en fait leur divergence la plus aiguë. L'EOKDE (Organisation unifiée des communistes internationalistes de Grèce, section de la Quatrième Internationale), le groupe de Pouliopoulos, continuait de considérer, dans la tradition trotskyste, l'Union soviétique comme un «État ouvrier dégénéré ", et donc, il était pour sa défense militaire inconditionnelle contre l'impérialisme capitaliste. Le groupe de Stinas, au contraire, refusait de prendre le parti de l'URSS. Un débat similaire fit rage dans de nombreuses sections trotskystes, notamment aux USA, où il y eut une scission majeure en 1940 sur cette question.

Stinas ne rejeta pas immédiatement la caractérisation théorique de l'URSS comme «État ouvrier dégénéré ». Mais il la rejeta en pratique en renonçant à la défense de l'URSS, arguant que cela reviendrait à défendre le camp impérialiste dans lequel se trouvait l'URSS dans le conflit mondial. À partir de 1941, cela voulait dire le camp des Alliés, mais au moment où Stinas lance sa grande polémique sur la question de l'URSS, en octobre 1940, l'URSS était du côté de l'Axe fasciste, alors que Metaxas et une bonne partie de la bourgeoisie grecque s'étaient rangés du côté britannique. À ce moment, la pression ambiante en Grèce allait donc dans le sens de la condamnation de l'URSS qui se trouvait du côté opposé à celui de la bourgeoisie grecque.

Stinas considérait la «bureaucratie stalinienne » comme le danger principal en Union soviétique, plus grand encore que le danger d'une contre-révolution capitaliste. La lutte pour renverser la bureaucratie stalinienne était donc pour lui la tâche essentielle concernant l'URSS, même si elle devait aboutir à la restauration du capitalisme. L'EOKDE était fermement contre cette position et maintenait l'orthodoxie trotskyste sur la défense inconditionnelle de l'URSS. Lorsque, à partir de 1942, les organisations trotskystes recommencèrent à diffuser des publications clandestines, l'EOKDE continua d'insister sur la défense de l'URSS, dénonçant la KDEE de Stinas sur cette question et disant que, pour cette raison, la KDEE se plaçait en dehors de la Quatrième Internationale. 
19 L'EOKDE défendait l'URSS, tout en prônant une révolution politique ouvrière contre la bureaucratie et en mettant en garde sur l'Armée rouge qui avait été transformée, disait-elle, en organe d'autoconservation et de satisfaction des rêves et désirs de la bureaucratie stalinienne. L'EOKDE luttait par ailleurs contre les illusions dans l'impérialisme "démocratique " des USA et de l'Angleterre et prévenait que, s'il arrivait en Grèce, ce ne serait pas pour libérer le peuple grec.

Elle prônait la fraternisation avec les soldats conscrits dans les armées allemande et italienne, ce qui contrastait avec la propagande des staliniens, complètement alignés sur le camp allié et hostiles à l'ensemble des troupes d'occupation, de haut en bas. Quand l'EOKDE parle "d'oppression nationale ", elle le fait dans un cadre général, jamais comme une oppression liée à l'occupation de la Grèce à ce moment-là. Elle défend le droit à l'autodétermination, pouvant aller jusqu'à la séparation, de toutes les nationalités opprimées, y compris en Épire, en Macédoine et en Thrace. Elle dénonce les attaques du KKE contre les femmes qui avaient des relations sexuelles avec des soldats faisant partie des troupes d'occupation.

21 L'EOKDE expliquait que, sur le fond, les intérêts de la bourgeoisie grecque coïncidaient avec ceux des Alliés plutôt qu'avec l'Axe, malgré la pénétration économique allemande avant la guerre, car, en cas de victoire alliée, la bourgeoisie grecque pouvait espérer récupérer l'Épire et le Dodécanèse, alors qu'en cas de victoire de l'Axe, ce sont les Bulgares et les Italiens qui s'arrogeraient des territoires grecs.

L'EOKDE décrit l'EAM, le front de libération nationale dirigé par le KKE, comme inféodé à l'état-major britannique. En réalité, l'EAM a toujours conservé le contrôle de ses propres décisions militaires (jusqu'à l'accord de Caserte). Les Britanniques avaient bien des conseillers auprès de l'EAM, mais ce n'est pas eux qui décidaient en dernier ressort des opérations militaires de l'EAM. L'EOKDE a même écrit que l'EAM s'était mise à la disposition de l'EDES, l'organisation de partisans de Zervas, ce général ex-vénizéliste à la réputation sulfureuse qui disposait du soutien financier et militaire des Britanniques. C'est manifestement faux, l'EAM ayant même tenté de liquider physiquement les maquis pro-britanniques de l'EDES. Toutefois les trotskystes ont pris implicitement le côté de l'EAM contre l'EDES lors de ces combats à l'automne 1943.

\section{Les trotskystes et la Résistance}

Les trotskystes étaient globalement hostiles au mouvement de résistance dirigé par le KKE, y compris dans les villes, sur la base de l'opposition à la politique de collaboration de classe du parti communiste avec des bourgeois grecs dits progressistes et avec les impérialistes alliés. Les trotskystes publient ainsi des articles sur des grèves et protestations ouvrières, où, le cas échéant, ils s'attachent à dénoncer un patron "progressiste » qui se donne des prétentions anti-collaborationnistes. Par exemple à l'usine de cigarettes Papastratos au Pirée, la direction de l'usine essaie de désamorcer le mécontentement des ouvrières en arguant de son propre patriotisme avec «nos alliés Staline, Roosevelt et Churchill»; vu la réponse pleine de défiance des travailleuses, annonçant que c'est l'expropriation pure et simple des capitalistes qui sera à l'ordre du jour après la guerre, la direction envoie des gros bras pour disperser les ouvrières, en blessant plusieurs (journal L'Internationaliste de l'EOKDE, 6 octobre 1943). 
Malgré cela, certains trotskystes furent actifs dans les maquis de l'EAM. Par exemple Stavros Verouchis, qui fut élu au Conseil national du PEEA, le gouvernement provisoire issu de la résistance communiste, et qui fut assassiné en 1944 par les staliniens. Les archéiomarxistes, qui avaient rompu avec Trotsky à partir de 1933, avaient une attitude plus positive vis-à-vis de l'EAM que les trotskystes proprement dits. Certains rejoignirent l'EAM et beaucoup furent tués en 1944 par les staliniens du KKE qui ne voulaient pas voir leur contrôle politique sur l'EAM battu en brèche par quiconque. Certaines sources indiquent que Verouchis était archéiomarxiste quand il fut élu au conseil du PEEA ; quoi qu'il en soit, les frontières entre les différents groupes étaient souvent perméables et les gens passaient d'un groupe à l'autre assez facilement. Apparemment, lors de la conférence trotskyste de début 1944, il y a eu une lutte contre une minorité mieux disposée vis-à-vis de l'EAM, voire même guérilliste. Vérouchis représentait peut-être cette tendance lorsqu'il préconisait des maquis trotskystes.

À partir de l'automne 1942 les grèves et une agitation de masse commencèrent dans les villes, d'abord avec une grève de 60000 employés dans la région d'Athènes-Le Pirée. Le KKE en prit la direction, au grand dam des trotskystes (qui, on peut le supposer, n'appréciaient pas de se faire évincer de l'influence qu'ils pouvaient avoir parmi les travailleurs) qui qualifièrent cette grève de défaite, le KKE, au contraire, proclamant une grande victoire. Les trotskystes eurent du mal à reconnaître le renforcement de leurs adversaires ; ils étaient au contraire convaincus que le KKE était en pleine crise et qu'il se trouvait écartelé entre d'un côté le nationalisme bourgeois pur et simple et, de l'autre côté, des courants centristes évoluant vers la gauche. Ils imaginaient donc qu'ils seraient eux-mêmes en position de rapidement gagner l'hégémonie sur les masses prolétariennes des centres urbains. Ces courants centristes dans le KKE étaient en réalité fantomatiques. L'EOKDE avait de trop faibles forces pour réellement savoir ce qui se passait et pour intervenir dans des débats éventuels dans les organisations contrôlées par le KKE.

L'occupant nazi tenta de faire appel au volontariat pour recruter des travailleurs grecs pour les usines du Reich, les travailleurs allemands se trouvant eux-mêmes mobilisés sur le front russe. Ce fut un échec. Quand, début 1943, l'occupant passa à la mobilisation forcée, l'équivalent du Service du travail obligatoire (STO) en France, cela provoqua d'immenses mobilisations, avec des grèves et manifestations massives dans les villes, qui contraignirent les nazis à reculer. Cette énorme mobilisation était dirigée par le KKE, quelques semaines après la victoire soviétique de Stalingrad qui fut la première défaite majeure de la Wehrmacht.

Les trotskystes grecs, en tout cas ceux de l'EOKDE, n'ont rien écrit sur la mobilisation contre le STO, malgré la dimension gigantesque que prit en Grèce la mobilisation ouvrière contre cette réquisition, de loin la plus importante qui ait eu lieu dans toute l'Europe occupée par l'Allemagne. Dans d'autres pays, les trotskystes avaient pour politique de ne pas s'opposer au STO ; au lieu de se cacher, comme le faisaient beaucoup de jeunes en France par exemple, des trotskystes, français et hollandais notamment, se sont laissé mobiliser pour l'Allemagne dans le but de faire la jonction avec le prolétariat allemand qui, à leurs yeux, allait jouer le rôle décisif à la fin de la guerre.

Il est possible que les trotskystes grecs aient été embarrassés sur l'attitude à adopter par rapport à la mobilisation ouvrière en Grèce contre le STO. En tout cas, entre février et septembre 1943, ils ne publient qu'un seul numéro de leur journal L'Internationaliste (daté de juin), exclusivement consacré à la mémoire de Pouliopoulos, exécuté par un 
officier italien en juin 1943; on raconte que Pouliopoulos, qui était polyglotte, s'était adressé en leur langue aux soldats italiens du peloton d'exécution, et qu'en conséquence, l'officier italien avait dû l'exécuter lui-même ; typiquement, l'exergue du numéro de L'Internationaliste consacré à Pouliopoulos appelle à la fraternisation avec les soldats italiens et allemands. Les trotskystes font porter la responsabilité de ce meurtre à égalité sur les autorités d'occupation et la bureaucratie "stalinienne » du KKE, car cette dernière avait refusé d'organiser l'évasion en masse des prisonniers du camp d'Acronauplie lors de l'effondrement de l'armée grecque face à la Wehrmacht début 1941 ; Pouliopoulos était donc resté prisonnier jusqu'à son exécution (alors qu'en fait, un certain nombre de dirigeants du KKE purent s'échapper).

A partir de fin 1943, les trotskystes parviennent à publier un ou deux journaux par mois. On y trouve quelques articles intéressants sur des grèves, notamment dans des usines au Pirée. Ils critiquent l'organisation militaire du KKE et lui opposent une « lutte de masse ", ce qui ne veut pas dire grand-chose dans le contexte du régime totalitaire d'occupation; les trotskystes préconisaient le développement d'organes de lutte avec des représentants élus. En fait, la liquidation physique de délateurs dans les usines par des groupes armés liés au KKE semble avoir eu un effet dissuasif dans certains cas et offert une protection aux travailleurs. Le problème n'est pas que les «staliniens » aient eu des groupes armés clandestins, mais qu'ils étaient étroitement contrôlés politiquement par le KKE.

Parfois les prises de positions des trotskystes frisent le "crétinisme démocratique », en pleine terreur policière, comme lorsqu'ils demandent la mise en place de coopératives pour la distribution de nourriture, avec un congrès démocratique pour discuter la situation, avec des prêts à taux zéro des banques, etc. À noter toutefois la participation des trotskystes, notamment ceux du groupe Stinas, à des expropriations de magasins et d'entrepôts, opérées en action commando avec distribution à la foule des produits saisis.

Les trotskystes ont bruyamment dénoncé le bombardement des quartiers ouvriers du Pirée par l'aviation alliée début 1944, qui, pour eux, était une preuve nouvelle de ce que les travailleurs pouvaient attendre d'un débarquement allié. En juin 1944, ils mettent en garde contre le débarquement allié en Normandie, disant qu'il a pour raison d'être de noyer dans le sang toute tentative de révolution ouvrière. Ils dénoncent aussi, en novembre 1943, la répression coloniale en Syrie-Liban opérée par les troupes gaullistes. Donc pendant toute la période, ils reviennent avec insistance sur la nécessité de ne pas avoir d'illusions dans les Alliés.

Début 1944, le KKDE (Parti des communistes internationalistes de Grèce section de la Quatrième Internationale - le nouveau nom de l'EOKDE) décide, lors de sa conférence, de mettre en sourdine les polémiques avec le DKKE de Stinas sur la nature de l'URSS, dans une perspective d'unification des deux groupes (qui n'aura pas lieu avant 1946). Le KKDE exige seulement de Stinas qu'il accepte la ligne majoritaire de la Quatrième Internationale en attendant une nouvelle discussion internationale. Toutefois l'EOKDE continue d'insister, dans presque chacune de ses publications, sur la défense de l'Union soviétique. À noter que, lors de son congrès, le KKDE retrace sa propre histoire au travers de la trajectoire de Pouliopoulos, en d'autres termes, il rejette toute l'histoire de l'archéiomarxisme qui, pourtant, avec constitué, au début des années 1930, la section officielle de l'Opposition de Gauche Internationale trotskyste, contrairement au groupe de Pouliopoulos. 

débarquement britannique au Pirée. Ils dénoncent le gouvernement d'unité nationale, où est représenté le KKE, comme un gouvernement bourgeois. Mais, à aucun moment, ils ne s'opposent explicitement aux troupes britanniques. Pourtant, on n'est pas comme en 1940 où la lutte contre l'occupation italienne revenait à se ranger dans le camp de Churchill et de Metaxas dans la guerre : cette fois-ci la bourgeoisie grecque dépend, pour sa survie, des troupes britanniques.

Les trotskystes sont optimistes et croient que la fin imminente de la guerre va ouvrir des situations révolutionnaires partout en Europe. Ils croient même qu'elles se 
développent déjà à Paris, et même à Hambourg. Ils prévoient que les communistes vont être un des plus solides piliers du rétablissement de l'ordre capitaliste, mais, du coup, ils n'anticipent pas la guerre civile qui va éclater un mois plus tard dans les rues $\mathrm{d}$ 'Athènes.

Ils avancent des revendications économiques et la nécessité du contrôle ouvrier, contre les mesures d'austérité du gouvernement dont fait partie le KKE. À la lecture de leur presse, notamment pour l'anniversaire de la révolution russe le 7 novembre, on peut voir qu'ils s'attendent à une crise révolutionnaire imminente. Quand éclate la guerre civile en décembre 1944, il semble qu'ils aient cherché à prendre part aux combats aux côtés de l'EAM. Mais beaucoup ont été tués par les staliniens de l'OPLA (milice politique du KKE), et ils ont dû probablement passer plus de temps à essayer de se protéger du parti qu'à combattre les troupes britanniques et grecques; ils étaient trop faibles pour produire de la propagande dans une telle situation de guerre civile ouverte.

En février 1945 ils adoptent une résolution décrivant les événements de décembre comme un conflit entre des forces sociales ennemies où, derrière le KKE, se trouvaient les couches populaires et les masses opprimées et, derrière le camp opposé, le capital. Ils voyaient que le KKE n'avait pas pour but la prise révolutionnaire du pouvoir, mais seulement la revendication de démocratiser l'État et de réforme politique dans le cadre bourgeois, ce qui (selon les études de nombreux historiens) est exact.

Dans leur bilan, ils ne prennent pas le parti du KKE dans les affrontements de décembre 1944. Ils insistent sur les « actions criminelles et barbares » des « staliniens » plutôt que sur celles des troupes britanniques et des tueurs anticommunistes de la bourgeoisie grecque. Ils parlent d'une trahison dans la lignée de l'Allemagne en 1933, quand les communistes laissèrent Hitler prendre le pouvoir sans coup férir. Sauf qu'à Athènes, il y a eu bataille.

42 Les trotskystes américains au contraire, qui étaient à l'époque la section la plus importante et la plus stable de la Quatrième Internationale, ont clairement pris le côté des combattants de l'EAM dans leur journal, dénonçant les communistes staliniens non pour leur combat à Athènes, mais pour leur coalitionnisme antérieur, à Téhéran, Beyrouth et surtout Caserte, où ils avaient accepté de se subordonner aux troupes britanniques et de prendre part au gouvernement du Caire. Les trotskystes américains critiquent le KKE quand celui-ci s'excuse servilement après le massacre policier du 3 décembre de ne pas avoir rendu leurs armes, au lieu de sonner la mobilisation générale. Et, en février 1945, ils l'attaquent à nouveau pour ses offres constantes de capitulation, y compris l'acceptation d'un régent. C'est un ton très différent des trotskystes grecs. En quelque sorte, les trotskystes grecs critiquent le KKE pour l'insurrection de décembre 44 contre les impérialistes britanniques et les capitalistes grecs, les trotskystes américains le critiquent pour les accords de Varkiza (où il accepte de rendre les armes aux Britanniques et au gouvernement capitaliste grec).

En 1946, Georges Vitsoris, qui travaillait avec Michel Pablo, un autre militant d'origine grecque, dans le secrétariat international trotskyste à Paris, écrivit une lettre qui devait servir de cadre politique à la fusion entre les groupes trotskystes grecs. La lettre critiquait vertement leur "sectarisme" pendant la guerre et leur incapacité à intervenir dans le mouvement de résistance; elle exagérait le caractère prolétarien de la résistance et allait dans le sens d'une adaptation politique au stalinisme (quelques années plus tard, Pablo allait ordonner la liquidation des sections trotskystes, pour entrer à long terme dans différentes formations suivant le terrain national, mais 
surtout les partis communistes, comme en France). Mais elle touchait du doigt le fait qu'à cause de leur abstentionnisme durant cette période, de leur neutralité, ils s'étaient neutralisé eux-mêmes comme facteur pour polariser des organisations staliniennes pétries de contradictions. Ayant manqué de mener une lutte décisive pour arracher le pouvoir en décembre 1944, après la capitulation de Varkiza, les «staliniens " s'étaient déconsidérés auprès de certaines couches de militants politiques.

Fin 1946 les trotskystes eurent des débats publics avec des communistes, ce qui reflète qu'ils avaient une certaine assise politique les obligeant à débattre avec eux. Par exemple, il y eut un débat, le 3 novembre 1946, entre Stinas pour la Quatrième Internationale et Apostolou pour le KKE sur le thème "République populaire ou république soviétique en Grèce». La présentation de Stinas a été conservée et reproduite, par exemple dans Quatrième Internationale de mai-juin 1947. Évidemment, le KKE défend à l'époque "la révolution bourgeoise démocratique, et non pas la révolution socialiste, comme le disent les trotskystes. » En dépit du sujet qui se prêtait à faire un bilan de la résistance et de décembre 1944, Stinas est incapable de soulever ces questions brûlantes et, au lieu de cela, se livre à une apologie en termes très généraux de la dictature du prolétariat. Du coup, en réponse à une question, Apostolou peut se permettre de dire que "les évêques sont partis dans le maquis, alors que les trotskystes, sous le couvert de phrases gauchistes, étaient au service de Hitler. Le PCG [le KKE] a réalisé le front unique avec tous ceux qui luttent avec le peuple, et non pas avec ceux qui, sous une phrase gauchiste, empêchent le développement du mouvement populaire. »

Évidemment, on reconnaît le style classique de la calomnie anti-trotskyste des "staliniens ", mais la position des trotskystes pendant la guerre facilitait la tâche de leurs adversaires. Ils allaient préserver leur hégémonie parmi les éléments les plus radicaux du prolétariat grec.

\section{BIBLIOGRAPHIE}

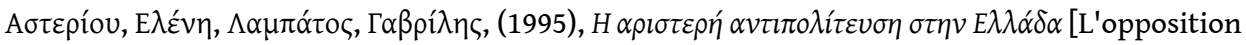

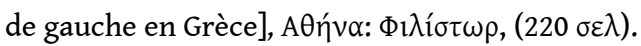

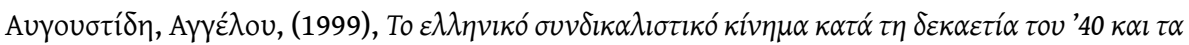
$\pi \varepsilon \rho \imath \theta \dot{\omega} \imath \imath \alpha \tau\rceil \varsigma \pi \lambda_{\imath \tau \imath \kappa \eta ́ \varsigma}$ [Le mouvement syndicaliste pendant la décennie 1940 et les marges de la

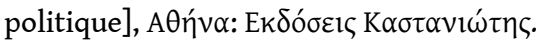

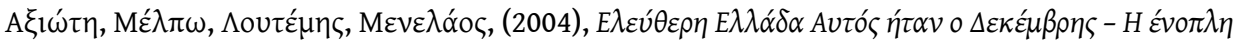

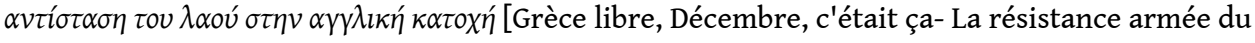

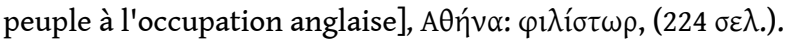

Baerentzen, Lars (éd.), (1982) British Reports on Greece by J.M. Stevens, C.M. Woodhouse \& D.J. Wallace, Copenhagen: Museum Tusculanum Press, (224 pages).

Chiclet, Christophe, (1987), Les communistes grecs dans la guerre, Paris : L'Harmattan, (324 pages). 


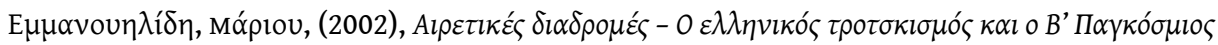

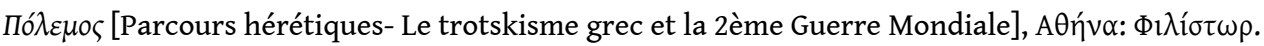

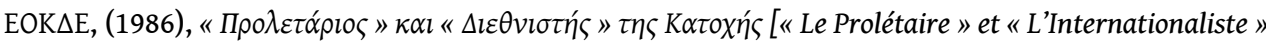

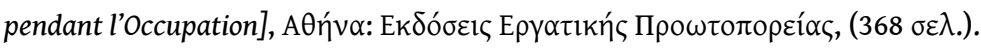

Eudes, Dominique, (1970) Les Kapetanios - La guerre civile grecque de 1943 à 1949, Paris : Fayard, (493 pages).

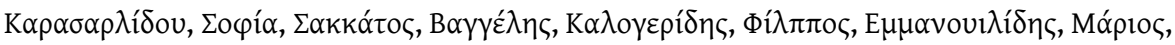

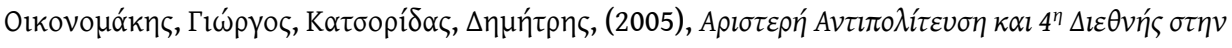

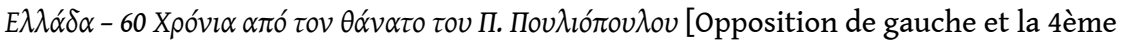

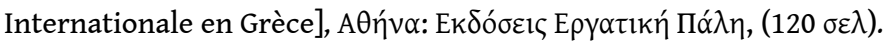

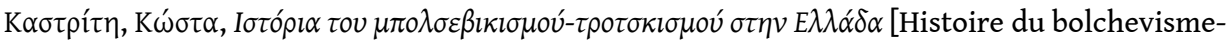

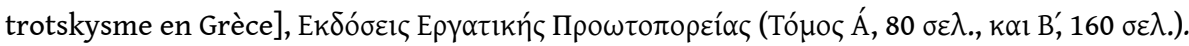

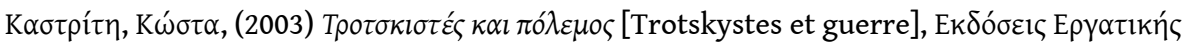

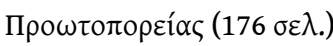

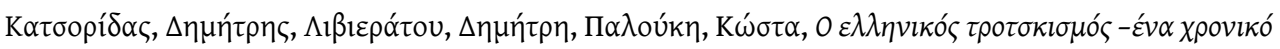

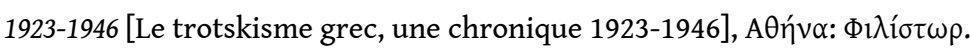

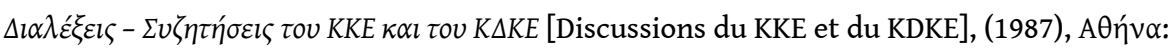

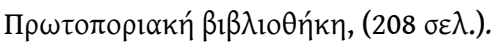

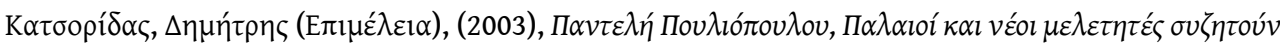

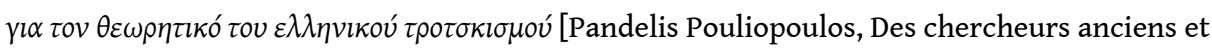

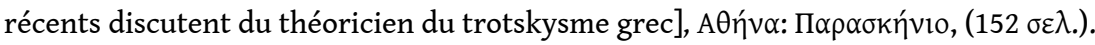

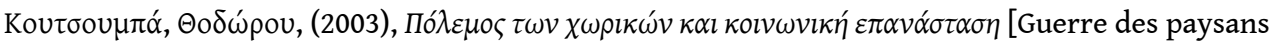

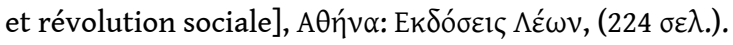

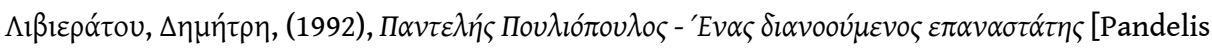

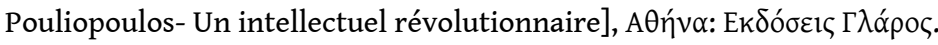

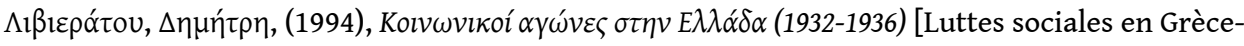

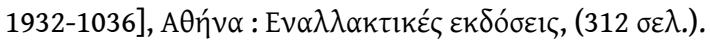

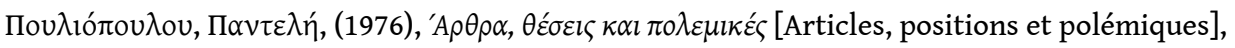

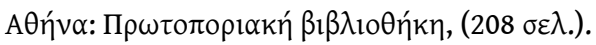

Prager, Rodolphe (éd.), (1981), Les congrès de la IVe Internationale - 2. L'internationale dans la guerre (1940-1946), Paris : La Brèche, (480 pages).

Prager, Rodolphe (éd.), (1988), Les congrès de la IVe Internationale - 3. Bouleversements et crises de l'après-guerre (1946-1950), Paris : La Brèche, (480 pages).

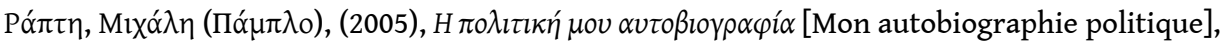

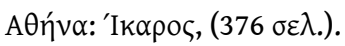

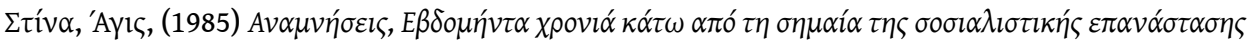
[Soixante-dix ans sous la bannière de la révolution socialiste], $A \theta \eta ́ v \alpha: ~ Y \psi \imath \lambda$ ov/ $\beta \imath \beta \lambda i ́ \alpha, \delta \varepsilon v ́ \tau \varepsilon \rho \eta$ $\varepsilon ́ \kappa \delta \circ \sigma \eta(512 \sigma \varepsilon \lambda$.).

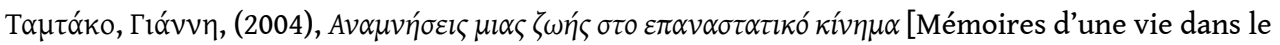

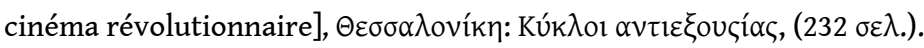




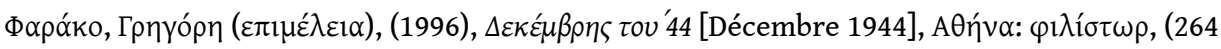
$\sigma \varepsilon \lambda$.$) .$

\section{Articles}

Cahiers Léon Trotsky $\mathrm{n}^{\circ}$ 76, décembre 2001.

Revolutionary History, vol. 3.3, printemps 1991, "Trotskyism and Stalinism in Greece"

\section{RÉSUMÉS}

Comment les deux courants du trotskysme grec, pourchassés dès les années 30, ont-ils réagi face à l'occupation de leur pays et à la résistance d'obédience communiste ?

Les trotskystes en Grèce étaient divisés, malgré des discussions d'unification dans les camps de Metaxas. Ils refusèrent de soutenir la Grèce contre l'intervention italo-allemande en 1940-1941 et s'opposèrent aux illusions dans les démocraties alliées. Fin 1942 ils reprirent une activité clandestine, refusant de se joindre à la Résistance mais aussi de choisir un côté militaire dans les affrontements avec l'occupant et ses supplétifs. En octobre 1944, les trotskystes s'agitèrent contre les Britanniques pendant que l'EAM les laissait entrer dans Athènes. Le KKE exécuta des dizaines de trotskystes. Ceux-ci restèrent neutres dans la guerre civile de décembre 1944. Leur sectarisme contribua à les stériliser lorsque, après les accords de Varkiza en février 1945, la direction du KKE était vulnérable aux accusations qu'elle avait conduit la révolution à la défaite.

Trotskyists in Greece were divided, despite unification discussions in Metaxas' concentration camps. They refused to support Greece against the 1940-41 Italo-German intervention and opposed illusions in Allied democracies. At the end of 1942 they resumed underground activity, refusing to join the Resistance but also to take a military side in the fighting against the occupiers and their local proxy forces. In October 1944 the Trotskyists agitated against the British while the EAM let them into Athens. The KKE executed dozens of Trotskyists. The Trotskyists remained neutral in the December 1944 civil war. Their sectarianism contributed to sterilize them when, after the February 1945 Varkiza Agreement, the KKE leadership was vulnerable to accusations of having led the revolution to defeat.

\section{INDEX}

Index géographique : Grèce, Athènes, Macédoine, Varkiza

motsclesmk АТИНА, ГРЦИЈА, МАКЕДОНИЈА

motsclestr Atina, Yunanistan, Makedonya, Varkiza, Dünya savaşı (1939-1945), Metaxas

diktatörlük (1936-1941)

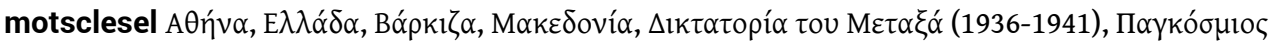

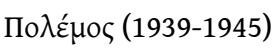

Index chronologique : guerre mondiale (1939-1945), dictature de Métaxas (1936-1941)

Thèmes : Histoire

Mots-clés : trotskysme grec, Pouliopoulos Pantelis (1900-1943), Archéiomarxistes, Bataillons de sécurité, archeiomarxistes, Caserte (accord de), Décembre 1944, EAM, EDES, EOKDE, KKDE, Kokkinia, OPLA, Pablo (1911-1996), Pangalos Théodore (1878-1952), PEEA, Pouliopoulos Pantelis (1900-1943), SEKE, Stinas Agis (1900-1987), Varkiza, Zachariadis Nikos (1903-1973), Acronauplie Keywords : Archeiomarxism, Greek trotskysm, Pouliopoulos Pandelis (1900-1943), Macedonia, History, Greece 
AUTEUR

ALEXIS HEN

Doctorant CREE-CEB EA 4513 